Chapter 9

\title{
Microscopic Interferometry by Reflection Holography with Photorefractive Sillenite Crystals
}

\author{
Eduardo Acedo Barbosa, \\ Danilo Mariano da Silva and Merilyn Santos Ferreira \\ Additional information is available at the end of the chapter \\ http://dx.doi.org/10.5772/53416
}

\section{Introduction}

The continuous growth of the development and production of micro- and nano-scaled devices requires the employ of compatible measurement techniques which fulfill the increasing demands of this new field. Despite the several very well established microscopy techniques, the potentialities of measuring the dynamic behavior of devices like microelectromechanical systems (MEMS) or capacitive micromachined ultrasonic transducers (CMUTs) were not sufficiently explored so far. In this framework, microscopic holographic interferometry and microscopic DSPI (digital speckle pattern interferometry) have been demonstrated to be very promising techniques for micro-scaled device measurement, due to its whole-field character, precision and accuracy. Due to its incomparable resolution and to the possibility of performing real-time testing with a minimum of computational image processing, photorefractive holography is a powerful tool for this task.

Photorefractive materials comprise a class of materials which present an interesting combination of physical properties. They combine light sensitivity with photoconductivity and the linear electrooptic effect. This effect was first observed in the years 1960 at Bell Laboratories $[1,2]$, in second harmonic generation experiments in ferroelectric crystals. At the time the effect was classified as "optical damage" by their observers, in function of localized variations in refractive index of the crystal with laser incidence.

Among the photorefractive crystals there are the ones from the sillenite family, namely: $\mathrm{Bi}_{12} \mathrm{GeO}_{20}$ (BGO), $\mathrm{Bi}_{12} \mathrm{SiO}_{20}(\mathrm{BSO})$, and $\mathrm{Bi}_{12} \mathrm{TiO}_{20}$ (BTO) [3, 4]. These crystals have a cubic lattice with symmetry group 23 , with only the elements $r_{14}, r_{52}$ and $r_{63}$ electro tensors being nonzero. They are optically active and become linearly birefringent in the presence of an electric field. 
As observed in other photorefractive materials working as holographic media, the sillenites have attractive characteristics for holographic applications such as infinite cycles of simultaneous, real-time holographic recording and reconstruction, all without fatigue or need of chemical processing.

The BTO crystal presents a lower optical activity for red light if compared to BSO and BGO, which makes it more appropriate for holographic applications, providing the highest possible diffraction efficiency for this wavelength range and enabling holography with the easily available and cost-effective He-Ne and red diode lasers. Its low diffraction efficiency can be compensated by its anisotropic diffraction properties [5, 6]. Due to this characteristic, under certain conditions the transmitted beam and diffracted beam propagate with orthogonal polarization at the crystal output, so that the signal-to-noise ratio of the output image can be increased by eliminating the beam transmitted by means of a linear polarizer.

All these features make this crystal an extremely interesting medium for many applications like real-time interferometry [7, 8, 9], spatial light modulation [10, 11, 12], nonlinear optical signal processing [13, 14], coherent image processing [15], edge detection [16], phase conjugation [17, 18], pattern recognition [19, 20], information storage [21] as well as phase conjugation in optical resonators [22]. Two-wave mixing with transmission holography configuration is the most employed arrangement with photorefractive sillenite crystals. However, reflection holography with these materials have been received relatively few attention, in spite of the fact that this sort of holographic configuration has been proved to be quite effective and powerful for interferometry and image processing [23].

The first study of a sillenite crystal for applications in interferometry was performed by Kukhtarev et al by using a [111]-cut BTO crystal and employing anisotropic diffraction in order to read the diffracted wave only [24]; the influence of the input polarization on the coupling of the interfering beams in reflection holograms with BTO and BSO crystals was developed by Mallick et al; Weber et al analyzed the gain and the diffraction efficiency as a function of the input polarization angle for BTO and BSO crystals with the grating vector parallel to the [001]-axis [25]. Salazar et al recorded a speckle pattern in sillenite crystals in a reflection holography configuration [26] in order to develop a four wave-mixing optical correlator through reflection holography [27]. Regarding recent applications of selenite crystals in reflection holography we can mention Erbschloe et al, which investigated the intensity and the phase of two optical beams in a photorefractive material, showing the implications of a reflection holography geometry [28]; Shepelevich et al investigated the dependence of maximum diffraction efficiency of volume reflection holograms recorded in BSO crystals [29]; Shandarov et al studied the interaction of two light waves in a network formed in a dynamic reflection holography scheme [30].

The lensless Denisiuk configuration [31] for reflection holography allows obtaining high resolution holographic images in very compact and simple setups, which is a particularly valuable characteristic for microscope construction. This optical conception consists in placing the studied object close to the holographic medium through which the image can be collected in a wide angular range, favoring the image resolution. A sillenite-based optical setup for reflection holography aiming practical purposes like holographic interferometry must basically combine the simplicity of such arrangement and the high image quality obtainable 
through the anisotropic diffraction properties of the sillenite crystals. In this framework, it has been proposed a novel optical display for holographic imaging and interferometry using a $\mathrm{Bi}_{12} \mathrm{TiO}_{20}$ crystal and red lasers as light source. This optical arrangement employed as a keyelement a polarizing beam splitter (PBS) positioned at the BTO input for polarization selection [23]. The potentialities of the setup for holographic imaging, double exposure holography and time average holography of small diffusely scattering objects are theoretically studied and experimentally demonstrated in this chapter. Aiming the development of a measurement device which allows the dynamic characterization of small components, this chapter analyzes the combination of three optical features: the Denisiuk configuration, the holographic recording and readout with sillenite crystals and the compound microscope geometry. The performance of the holographic microscope in the characterization of microscopic transducers and MEMs is described and potencial applications in the study of other micro-components are pointed out.

\section{Denisiuk setup with photorefractive crystals}

The Denisiuk configuration is the simplest geometry for reflection holography. The original setup comprises a light source, an object, and a recording medium between them. Since the object is usually positioned right behind the holographic medium, when both are illuminated the medium works also as a beam splitter: the laser beam hitting the front face of the medium is the reference wave, while the beam transmitted through the medium illuminates the object. The light scattered by the object illuminates the holographic medium through its rear face the object beam - and interferes with the reference wave, thus generating the hologram.

The typical low diffraction efficiency of the sillenite crystals make them not suitable for realtime holography in an original Denisiuk configuration, since the transmitted object beam is much more intense than the holographic image, leading to a very small signal-to-noise ratio (SNR). In transmission holography setups this low-diffraction efficiency limitation is usually overcome by exploring the anisotropic diffraction properties of those crystals: by properly setting the input polarization angle of the incoming waves with respect to the crystal [001]axis, the diffracted and transmitted waves emerging from the sample become orthogonally polarized [5,6]. Hence, a conveniently positioned analyzer can block the transmitted wave and enable the propagation of the diffracted wave only, which is the holographic reconstruction of the object. However, this arrangement cannot be made in reflection holography because a polarizer behind the crystal adjusted to eliminate the transmitted beam would block the reference beam also.

Due to the reasons above, Denisiuk-type reflection holography experiments using anisotropic diffraction in sillenite crystals require an unusual approach and a novel optical display, which should obey the following requirements often adopted for interferometry design in order to avoid spurious elliptical polarization and consequent losses in the hologram diffraction efficiency: a - the light beam propagation occurs in a plane parallel to the table top; $b$ - the beam polarization must be kept whether perpendicular or parallel to this plane, except when the 
light polarization undergoes a rotation inside the BTO crystal due to its optical activity; c - the hole setup must be simple and compact, in order to enable the construction of rugged and portable holography microscopes.

Those requirements are achieved by using a polarizing beam splitter (PBS) cube. With this configuration, anisotropic diffraction can be optimally observed by tilting the sillenite crystal with respect to the axis perpendicular to the table top. The tilting angle is selected in order to enable the diffracted wave to be orthogonally polarized with respect both to the incident reference wave and to the transmitted object wave. In such a configuration the signal-to-noise ratio is maximized.

The PBS cube separates the s-polarized (whose electric field is perpendicular to the plane of incidence) from the $p$-polarized (electric field parallel to the plane of incidence) components of the incident light and generates two linearly polarized beams propagating in mutually perpendicular directions. The s-polarized beam emerges from the PBS at a right angle, while the $p$-polarized is transmitted through it.

Figure 1 schematically shows a setup for reflection holography with a BTO crystal with thickness $d$ and a PBS cube as the polarizing component. The $x-y$ plane is parallel to the table top. The s-polarized light coming from the laser hits the PBS and is $90^{\circ}$ deviated, hitting the BTO crystal as the reference wave, whose propagation vector $\vec{k}_{R}$ is shown in figure 1 . Due to the crystal optical activity, the wave polarization is rotated by an angle $\rho d$ (counterclockwise rotation, looking towards the negative $X$-axis in fig. 1) where $\rho$ is the optical rotatory power. The beam transmitted through the crystal illuminates the object and is scattered by it, forming the object wave. This wave has a propagation vector $\vec{k}_{S}$, parallel to the positive $X$-axis, and is polarized at an angle $\rho d$ with respect to the z-axis. The object beam travels back through the crystal and again undergoes a polarization rotation of - $\rho d$, such that both reference and object beams present the same polarization orientation throughout the whole holographic medium and propagate in opposite directions. At the output (left side in fig. 1) of the BTO crystal there are two beams emerging with the same propagation vector: the transmitted object beam (with electric field $\overrightarrow{S^{\prime}}$ parallel to the $Z$-axis) and the diffracted reference beam, which in turn is the holographic reconstruction of the object wavefront (shown as the electric field $\vec{U}$ ). If the polarization directions of both beams are mutually orthogonal, or as close as possible to it, the hologram SNR is highest. This can be accomplished by properly rotating the crystal around its [100]-axis, which is parallel to the $X$-axis shown in figure 1 .

When the optimal angle between the the [001]-axis and the input reference beam polarization is achieved, the diffracted wave with amplitude $\vec{U}$ at the BTO output is p-polarized due to anisotropic diffraction, as shown in figure 2: while figure 2a shows the reference wave polarization $\vec{R}$ at the BTO crystal input $x=d$ ("solid" vector) and the sample output at $x=0$ ("dashed" vector), figure $2 \mathrm{~b}$ shows the object wave amplitude vector $\vec{S}$ ("dashed" vector) hitting the BTO crystal and the polarization states of the transmitted $(\vec{S})$ and the diffracted $(\vec{U})$ waves at $x=d$. Notice that $\vec{U}$ and $\vec{S}$ in figure $2 \mathrm{~b}$ are nearly orthogonal, which provides the condition of highest SNR. In the next sections the photorefractive recording and readout will be analyzed in order to allow these conditions to be achieved. 


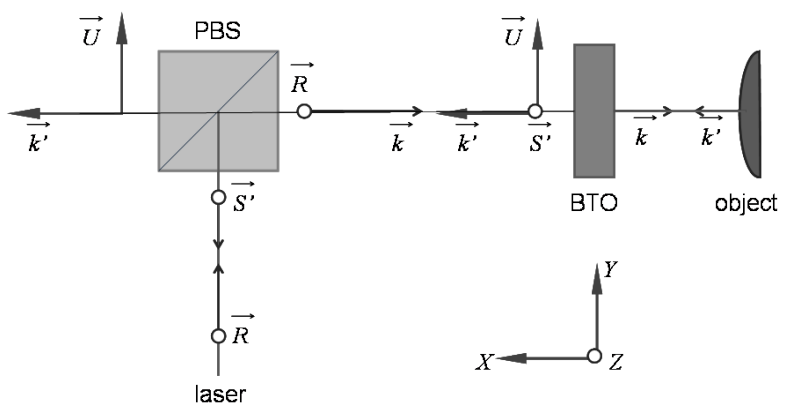

Figure 1. Sillenite-based Denisiuk holographic setup

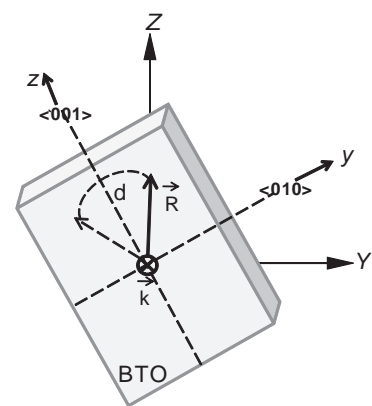

(a)

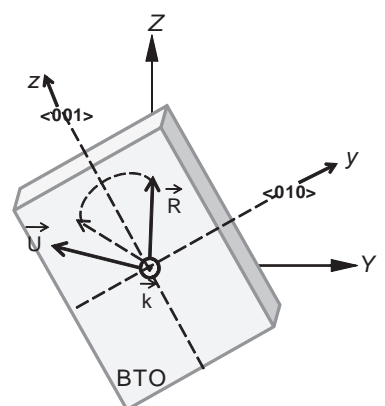

(b)

Figure 2. a - reference wave polarization $\vec{R}$ and its propagation vector $\vec{k}_{R}$ at the BTO input $x=d ; \mathrm{b}$ - $\operatorname{transmitted}\left(\vec{S}^{\prime}\right)$ and the diffracted $(\vec{U})$ beam polarizations propagating along positive $x$-axis at $x=d$.

\section{Photorefractive effect in sillenite crystals}

The photorefractive effect consists basically on the combination of two mechanisms: charge migration and electrooptic effect. In the former, charge carriers, say, electrons, are excited from occupied donor states to the conduction band due to the incidence of an interference pattern of light and then trapped by empty centers. In the latter, the resulting spatially non-uniform electric field due to the charge distribution modulates the refractive index, thus generating a phase index grating [3].

\subsection{Charge carrier migration}

Let us consider the incidence of a light interference pattern onto an impurity-dopped photorefractive crystal given by $=I_{0}(1+m \cos K x)$, where $K$ is the pattern spatial frequency and $m$ is the modulation due to the interacting beams intensities. In the literature several models describe the migration process [32], and among them, the band transport model has been more 
widely adopted. In the illuminated areas of the sample, photoelectrons from ionized donors indergoe a transition to the conduction band. In this band the electrons migrate by diffusion and/or drift, i. e., under the influence of an externally applied electrical field. After relaxation the electrons are captured by acceptor centers. This process of excitation to the conduction band and posterior recombination is repeated until the photoelectrons are trapped in dark regions of the sample (interference pattern minima). Correspondingly, holes can be excited from filled acceptors to the valence band.

In typical experimental conditions for holographic imaging, $I_{R} \gg I_{S}$, so that $m \ll 1$. In this case, the low-modulation interference pattern can be expressed in terms of the first harmonic of its Fourier expansion according to

$$
I=I_{0}+\frac{I_{0}}{2}\left(m e^{i K x}+m^{*} e^{i K x}\right)
$$

where $m=2\left(I_{S} I_{R}\right)^{1 / 2} e^{-t i \theta} /\left(I_{S}+I_{R}\right)$, and $\phi$ is the arbitrary phase of the signal (object) wave. The correspondent space charge electric field is written as

$$
E(x)=E_{0}+\frac{E_{S C}}{2} e^{i K x}+\frac{E_{S C}^{*}}{2} e^{i K x}
$$

Through Poisson equation $\operatorname{div}(\varepsilon \vec{E})=\rho / \varepsilon_{0}$, the continuity equation $\partial \rho / \partial t=-\partial j / \partial x$ and the equation for the current density $j=\mu e n(x)+e D \partial n / \partial x$, where $D$ is the diffusion coefficient and $n(x)$ is the electron concentration in the conduction band, the amplitude $E_{s c}$ of the steady-state space charge field in the holographic recording by diffusion $\left(E_{0}=0\right)$ is given by

$$
E_{S C}=\frac{-i m E_{D}}{1+K^{2} L_{S}^{2}}
$$

where $L_{s}$ is the Debye screening length and the $E_{D}$ is the diffusion electric field given in terms of Boltzmann's constant $k_{B}$ and the temperature $T$ as $E_{D}=K k_{B} T / e$.

\subsection{Electrooptic effect}

The propagation of the electromagnetic wave in a medium can be described with the help of the index ellipsoid, which provides the wave velocity according to its polarization state:

$$
\frac{x^{2}}{n_{x}^{2}}+\frac{y^{2}}{n_{y}^{2}}+\frac{z^{2}}{n_{z}^{2}}=1
$$

where $x, y$ and $z$ are the principal dielectric axes. If the medium is isotropic, $n_{x}=n_{y}=n_{z}$, and the light velocity does not depend on its polarization state in this case. 
When a crystal without inversion simmetry is under the influence of an electric field, there is a change in the index ellipsoid, now represented by

$$
\left(\frac{1}{n^{2}}\right)_{1} x^{2}+\left(\frac{1}{n^{2}}\right)_{2} y^{2}+\left(\frac{1}{n^{2}}\right)_{3} z^{2}+2\left(\frac{1}{n^{2}}\right)_{4} y z+2\left(\frac{1}{n^{2}}\right)_{5} x z+2\left(\frac{1}{n^{2}}\right)_{6} x y=1
$$

The terms $\left(1 / n^{2}\right)_{i}$ are determined as a function of an arbitrary electric field through the matrix relation

$$
\left(\frac{1}{n^{2}}\right)_{i}=\sum_{j=1}^{3} r_{i j} E_{j}
$$

where the electrooptic coefficients $r_{i j}$ are the elements of the $6 \times 3$ electrooptic tensor. By finding a new coordinate system $x^{\prime}, y^{\prime}, z^{\prime}$ through which equation (5) assumes the form of equation (4), the refractive index modulation along these axes can be determined. Since the symmetry group of the sillenite crystals is 23 and the only nonzero elements of the electrooptic tensor are $\mathrm{r}_{14}, \mathrm{r}_{52}$ and $\mathrm{r}_{63}\left(r_{41}=r_{52}=r_{63}=5 \times 10^{-12} \mathrm{~m} / \mathrm{V}\right.$ for BTO), the electrooptic tensor of such crystals is written as

$$
\left[\begin{array}{ccc}
0 & 0 & 0 \\
0 & 0 & 0 \\
0 & 0 & 0 \\
r_{41} & 0 & 0 \\
0 & r_{41} & 0 \\
0 & 0 & r_{41}
\end{array}\right]
$$

From relations (6) and (7) one obtains the coefficients for the crossed terms:

$$
\begin{aligned}
& \left(\frac{1}{n^{2}}\right)_{1}=r_{41} E_{X} \\
& \left(\frac{1}{n^{2}}\right)_{2}=r_{41} E_{Y} \\
& \left(\frac{1}{n^{2}}\right)_{3}=r_{41} E_{Z}
\end{aligned}
$$

Since $n_{x}=n_{y}=n_{z}=n_{0}$, the index ellipsoid expression takes the form

$$
\frac{x^{2}}{n_{0}^{2}}+\frac{y^{2}}{n_{0}^{2}}+\frac{z^{2}}{n_{0}^{2}}+2 r_{41} E_{S C} y z+2 r_{41} E_{S C} x z+2 r_{41} E_{S C} x y=1
$$


From equation (9) the refractive index modulation can be obtained, as it will be seen in detail in the next sections. Figure 3 summarizes the photorefractive mechanism, showing the incident light pattern (3a), the consequent steady-state charge distribution due to diffusion (3b), the space-charge electric field (3c) and the resulting refractive index modulation via electrooptic effect (3d). Notice that, in this case the electric field and the refractive index modulation are $\pi / 2$-shifted with respect with the light intensity pattern.

(a)

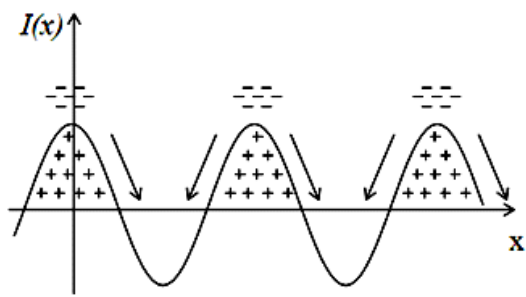

(b)

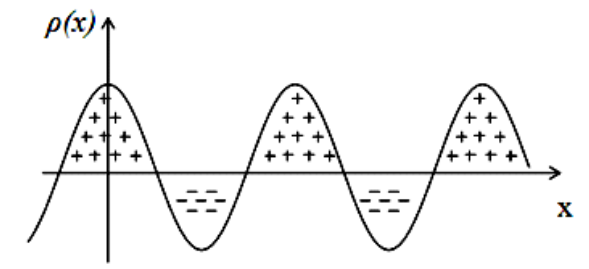

(c)

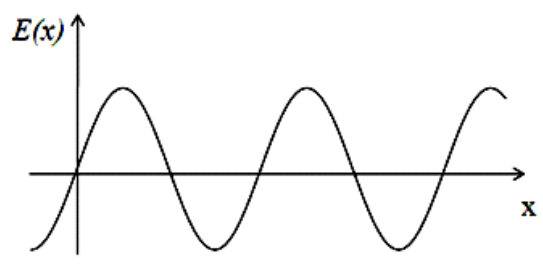

(d)

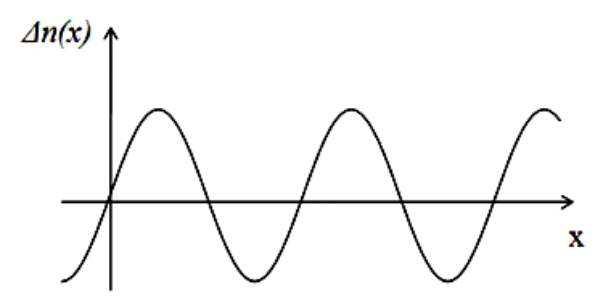

Figure 3. The photorefractive process; a - Intensity pattern; b - charge distribution; c-space-charge electric field and $d$-spatial refractive index modulation. 


\section{Reflection holography in photorefractive sillenite crystals}

\subsection{Recording}

In this section the holographic recording in a photorefractive sillenite crystal is studied considering the interference of two counterpropagating monochromatic coherent waves with wavelength $\lambda$. The resulting interference pattern rearranges the charge carriers by diffusion, leading to a spatially nonuniform electric field $E_{S C}$ inside the sample, as described in the previous section. Through the electrooptic effect this electric field gives rise to a phase grating due to local modulation of the refractive index. The crystal is cut in the transverse electrooptic configuration, in which its input facets are parallel to the crystalline (110) plane, as shown in figure 4 . Since the counterpropagating interfering waves (with propagation vectors $\vec{k}_{S}$ and $\overrightarrow{k_{R}}$ shown in this figure) incide normally to this facet, both the grating vector and the induced electric field are parallel to the [100]-axis, referred as to the $x$-axis in figure 4 . The index ellipsoid of equation (9) due to $E_{\mathrm{sc}}$ is then given by

$$
\frac{x^{2}}{n_{0}^{2}}+\frac{y^{2}}{n_{0}^{2}}+\frac{z^{2}}{n_{0}^{2}}+2 r_{41} E_{S C} Y Z=1
$$

where $n_{0}$ is the bulk refractive index. If the BTO crystal is rotated around the $X$-axis by an angle $\theta$, equation (10) can be conveniently expressed according to the coordinate system whose $x$-, $y$ - and $z$-axes are parallel to the principal crystalographic axes of the sample. In the rotation, $x$ and $X$ are parallel, so that the transformation relations are given by

$$
X=x ; \quad Y=y \cos \theta-z \sin \theta ; Z=y \sin \theta+z \cos \theta
$$

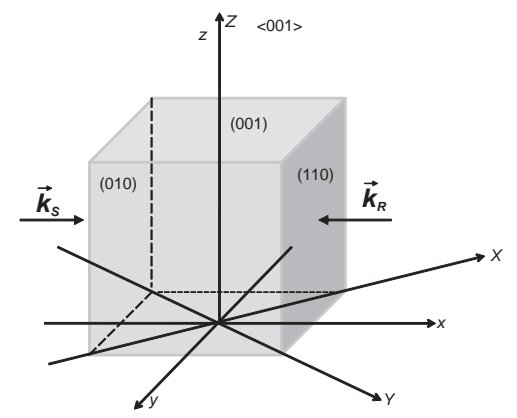

Figure 4. Electrooptic transverse-cut BTO crystal

By substituting $Y$ and $Z$ from eq. (11) into eq.(10), the index ellipsoid in the $x, y, z$ system is written as 


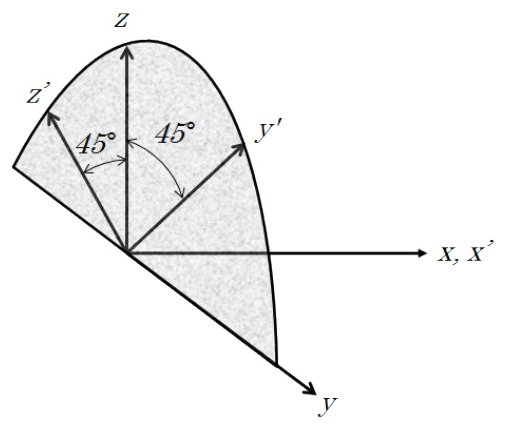

Figure 5. Rotation of the coordinate system around the $x$-axis

$$
\frac{x^{2}}{n_{0}^{2}}+\frac{y^{2}}{n_{0}^{2}}+\frac{z^{2}}{n_{0}^{2}}+2 r_{41} E_{S C} y z=1
$$

Notice that eqs. (10) and (12) have the same form, regardless the rotation angle $\theta$. The ellipsoid major axes $x^{\prime}, y^{\prime}, z^{\prime}$ can be determined by performing another coordinate transformation by a $45^{\circ}$-rotation around the $x$-axis, as shown in figure 5 , such that

$$
x=x^{\prime} ; y=y^{\prime} \cos 45^{\circ}-z^{\prime} \sin 45^{\circ} ; z=y^{\prime} \sin 45^{\circ}+z^{\prime} \cos 45^{\circ}
$$

Hence, upon substitution in eq. (12) this coordinate transformation yields

$$
\frac{x^{\prime 2}}{n_{0}^{2}}+y^{\prime 2}\left(\frac{1}{n_{0}^{2}}+r_{41} E_{S C}\right)+z^{\prime 2}\left(\frac{1}{n_{0}^{2}}+r_{41} E_{S C}\right)=1
$$

which corresponds to the following ellipsoid with $x^{\prime}, y^{\prime}$ and $z^{\prime}$ axes:

$$
\frac{x^{\prime 2}}{n_{0}^{2}}+\frac{y^{\prime 2}}{n_{y^{\prime}}^{2}}+\frac{z^{\prime 2}}{n_{z^{\prime}}^{2}}=1
$$

By comparing eqs. (14) and (15) one obtains

$$
\frac{1}{n_{y^{\prime}}^{2}}=\frac{1}{n_{0}^{2}}+r_{41} E_{S C} ; \quad \frac{1}{n_{z^{\prime}}^{2}}=\frac{1}{n_{0}^{2}}+r_{41} E_{S C}
$$

With the help of relation $d\left(1 / n^{2}\right)=-2 / n^{3} d n$ equation (16) provides the refractive index modulations along $\mathrm{y}^{\prime}$ and $\mathrm{z}^{\prime}$ axes:

$$
\Delta n_{y^{\prime}}=-\frac{n_{0}^{3}}{2}+r_{41} E_{S C^{\prime}} ; \Delta n_{z^{\prime}}=-\frac{n_{0}^{3}}{2}+r_{41} E_{S C}
$$


The results above evidence an anisotropy in the photorefractive phase gratings generated in the sillenite crystals, since $\Delta n_{y^{\prime}} \neq \Delta n_{z^{\prime}}$, which will be analysed in the next section.

\subsection{Readout; anisotropic diffraction}

In this section the experiment to confirm the theoretical predictions on the holographic process is performed by using a BTO crystal as holographic medium and a He-Ne laser as light source. In the setup the PBS is used in order to separate the diffracted and the transmitted beams and simultaneously allow the incidence of the reference wave onto the crystal. The intensity of the diffracted wave was measured as the BTO crystal was rotated, so that the dependence of the diffracted wave on the direction of the incident light polarization with respect to the crystal (001)-axis was studied. The experimental results very satisfactorily agreed with the theoretical analysis, showing that there is an optimal input polarization configuration through which the background noise generated by the transmitted object beam can be totally eliminated by the PBS, thus enabling the detection of the holographically reconstructed signal wave only [33]. From the theory presented in the previous section the amplitude of the wave diffracted by a reflection hologram in a self-diffraction process can be obtained. Due to the refractive index modulation anisotropy, the diffracted wave presents two different orthogonal components. This is the most important consequence of anisotropic diffraction. The dependence of the angle between the diffracted and the transmitted waves - a feature of crucial importance in this work - will be both theoretically and experimentally studied. In this section the angle between both polarization states can be theoretically obtained and the conditions to provide the best holographic image visibility will be analyzed.

The reference wave polarization at the BTO input with respect to the coordinate systems involved and the rotation angle $\gamma$ is shown in figure 6 . The components of the reference wave relatively to the $y^{\prime}$ - and $z^{\prime}$-axis are written as

$$
R_{y^{\prime}}(x)=R_{0} \cos [\theta-\rho(d-x)] ; \quad R_{z^{\prime}}(x)=R_{0} \sin [\theta-\rho(d-x)]
$$

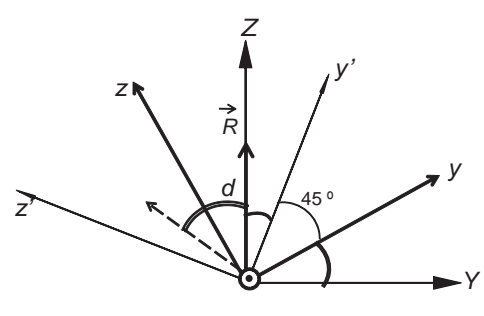

$X=x=x^{\prime}$

Figure 6. Reference wave polarization at the BTO input and the coordinate systems $X-Y-Z, x-y-Z$ and $x^{\prime}-y^{\prime}-Z^{\prime}$.

where $\theta$ is the angle between the reference wave polarization direction and the $y^{\prime}$-axis at the crystal output and $R_{0}$ is the amplitude of the reference beam at $x=0$. As mentioned earlier, the 
object beam and the reference beam polarizations are parallel throughout the crystal. The fringe contrast $m$ as a function of the crystal depth $x$ takes into account the changes of the interacting beams as they propagate along the sample [25]:

$$
m(x)=m_{0} \exp \left\{2 \frac{\beta}{\rho} \cos [2 \theta-\rho(2 d-x)] \sin (\rho x)\right\}
$$

where $\beta \equiv \pi r_{0}^{3} r_{41} E_{S C} / 2 \lambda$ and $m_{0} \approx 2 S_{0} / R_{0}$ for $R_{0} \gg S_{0}$, where $S_{0}$ is the object beam amplitude at $x=0$. The elementary components of the diffracted amplitude at $x=d$ are given by [25]

$$
d U_{y^{\prime}}(x)=-\beta m(x) R_{0} \cos (\theta-2 \rho d+\rho x) d x ; d U_{z^{\prime}}(x)=-\beta m(x) R_{0} \sin (\theta-2 \rho d+\rho x) d x
$$

where the sign of $d U_{y^{\prime}}(x)$ is due to the negative sign of $\Delta n_{y^{\prime}}$ in equation (17). Since $m_{0} \ll 1$ and $|\beta / \rho|<0.4, m(x)$ can be expanded into a Taylor series, so that from equation (19) one obtains

$$
m(x)=m_{0} \exp [-\beta / \rho \sin (2 \theta-2 \rho d)][1+\beta / \rho \cos (2 \theta-2 \rho d+2 \rho x)]
$$

By substituting $m(x)$ from equation (21) into equation (22) and integrating along the crystal thickness, the components of the diffracted amplitudes parallel to $y^{\prime}$ and $z^{\prime}$ are written as

$$
\begin{aligned}
& U_{y^{\prime}}(d)=-\beta R_{0} m_{0} \exp [-2 \beta \sin (2 \theta) / \rho] \times\left\{\frac{\sin \rho d}{\rho} \cos (\theta-\rho d)+\frac{\beta}{8 \rho^{2}}[\sin (4 \rho d-3 \theta)+\sin (3 \theta)+4 \rho d \cos \theta]\right\}(\mathrm{a}) \\
& U_{z^{\prime}}(d)=-\beta R_{0} m_{0} \exp [-2 \beta \sin (2 \theta) / \rho] \times\left\{\frac{\sin \rho d}{\rho} \cos (\theta-\rho d)+\frac{\beta}{8 \rho^{2}}[\cos (4 \rho d-3 \theta)-\cos (3 \theta)-4 \rho d \sin \theta]\right\}(b
\end{aligned}
$$

At the front face of the crystal the $y^{\prime}$ and $z^{\prime}$ components of the object wave amplitude are given by $S_{y^{\prime}}(d)=S_{0} \cos \theta$ and $S_{z^{\prime}}(d)=S_{0} \sin \theta$, respectively. Since this wave does not carry any relevant information and constitutes a noise to the holographically reconstructed wavefront, it is worthy cutting off the transmitted object beam by a polarizing element in order to provide the diffracted wave only. The maximal holographic signal $I_{U}$ corresponding to the holographic $\underset{\rightarrow}{i m a g e}$ must then be proportional to $\sin ^{2} \varphi$, where $\varphi$ is the angle between the vectors $\vec{U}=U_{y^{\prime}}(d) \hat{y}^{\prime}+U_{z^{\prime}}(d) \hat{z}^{\prime}$ and $\vec{S}=S_{y^{\prime}}(d) \hat{y}^{\prime}+S_{z^{\prime}}(d) \hat{z}^{\prime}$ :

$$
I_{U} \propto \sin ^{2} \sin ^{2} \varphi=1-\left(\frac{\vec{U} . \vec{S}}{|\vec{U}||\vec{S}|}\right)^{2}
$$

It is convenient to make the substitution $\theta=\pi / 4-\gamma$, where $\gamma$ is the angle between the [001]axis and the Z-axis, as shown in figure 6. With the help of equations (22a), (22b) and (23) one obtains the signal $\mathrm{I}_{\mathrm{U}}$ : 


$$
I_{U} \propto \sin ^{2} \varphi \approx 1-\frac{\left\{\sin (\rho d) \sin (2 \gamma+\rho d)+\frac{\beta}{8 \rho}[\sin 4 \gamma-\sin (4 \rho d-4 \gamma)+4 \rho d]\right\}^{2}}{\sin ^{2}(\rho d)+\frac{\beta}{4 \rho} \sin (\rho d)[4 \rho d \sin (2 \gamma+4 \rho d)+\cos (2 \gamma-\rho d)-\cos (2 \gamma+3 \rho d)]}
$$

For typical diffusely scattering objects, $\beta \ll \rho$, so that the signal $I_{U}$ from equation (10) can be satisfactorily written as

$$
I_{U} \approx \cos ^{2}(2 \gamma+\rho d)
$$

Through equation (25) the optimal angle between the crystal [001]-axis and the input polarization can be obtained to be $\gamma \approx-\rho d / 2+N \pi / 2$, where $N=1,2,3, \ldots$. In practical terms, since the polarization of the incident beam is perpendicular to the table top, the crystal must be tilted such that its [001]-axis makes an angle of $\gamma \approx-\rho d / 2$ with respect to the Z-axis, as shown in figure 7 .

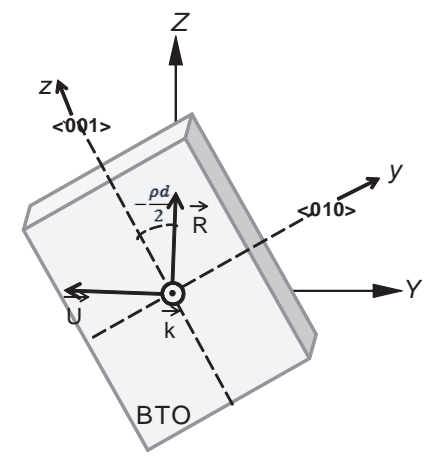

Figure 7. Optimal BTO orientation for maximum holographic image SNR.

\section{Experiments}

\subsection{Dependence of the diffracted beam intensity on the crystal tilting}

The intensity of the holographically reconstructed object beam as a function of the tilting angle $\gamma$ is investigated in order to confirm the theoretical prediction of equation (25). Figure 8 shows the optical setup for this purpose. The setup was illuminated by a 20-mW He-Ne laser emitting at $632.8 \mathrm{~nm}$. The s-polarized reference beam coming directly from the laser is expanded by lens L1. The crystal is cut in the electrooptic transverse configuration. The BTO sample is rotated around the $X$-direction by a goniometer. The transmitted object beam wave is deviated by the PBS, while the diffracted beam passes through it to be sent to the photodetector PD through lens L2. The sensitive area of the photodetector is larger than the diffracted beam cross 
section. The rotatory power of $\mathrm{Bi}_{12} \mathrm{TiO}_{20}$ is $\rho=-0.11 \mathrm{~mm}^{-1}$ for $632.8 \mathrm{~nm}$. For $m_{0} \approx 0.1$ and $n_{0} \approx 2.6, \beta$ was estimated to be $\sim 10^{-2} \mathrm{~mm}^{-1}$.

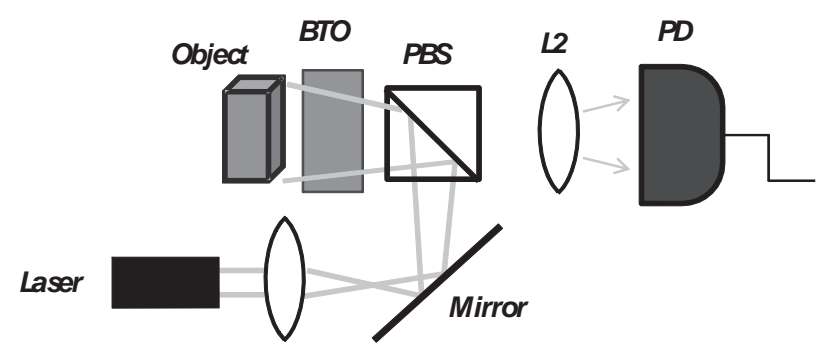

Figure 8. Optical setup: L1 and L2, lenses, M, mirror, PD, photodetector

Two BTO samples with sizes $10 \times 12 \times 10 \mathrm{~mm}^{3}$ (BTO 1), and $8 \times 7 \times 7 \mathrm{~mm}^{3}$ (BTO 2) were used. A $10 \times 10-\mathrm{mm}^{2}$ flat metallic bar placed right behind the crystal was used as the opaque scattering object surface. Figure 9a shows the dependence of $I_{U}$ on the orientation of the BTO 1 [001]-axis. The solid curve is the fitting of the experimental data with equation (25), showing maximum $I_{U}$ values for $\gamma \cong 30^{\circ}$ and $120^{\circ}$, showing a very good agreement with the optimal values theoretically obtained for the sample 1 thickness $(10 \mathrm{~mm})$ and the crystal rotatory power. Figure $9 \mathrm{~b}$ shows the same measurement for the 8-mm thick BTO 2 sample. The hologram buildup time was $\tau \sim 15$ s for both cases. Those measurements confirm the theoretical predictions and provide information to an optimal configuration in order to achieve holographic images with the best signal-to-noise-ratio.

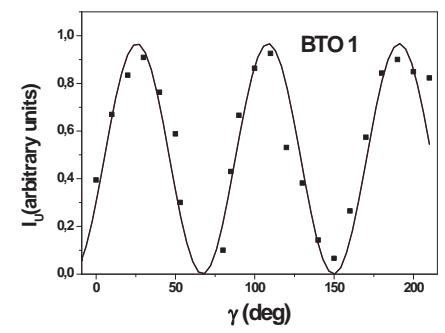

(a)

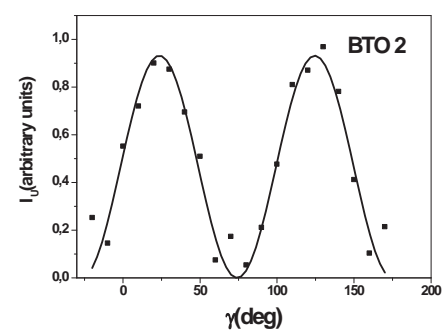

(b)

Figure 9. Signal $I_{U}$ as a function of the orientation angle $\gamma$; dotted curve, measured values; solid curve, fitting with equation (10), for a - BTO 1 and b-BTO 2 samples [23].

\subsection{Denisiuk-type reflection holography configurations for microscopy and interferometry}

In this section two geometries for reflection holography microscopes using a BTO crystal cut in the electrooptic transverse configuration for the characterization of microscopic devices are 
studied and analyzed. The polarizing beam-splitter did enable blocking the transmitted object beam and reading the diffracted wave, thus allowing for the reconstruction of the holographic image of the object in quasi real-time processes.

\subsubsection{First optical configuration}

In this optical setup a red diode laser emitting at $660 \mathrm{~nm}$ was employed as light source. A variation of the original Denisiuk optical scheme was developed by positioning the objective lens between the object and the holographic medium, so that the lens is used both to illuminate the object and to build its real image in front of the ocular lens. The beam coming from the laser is slightly expanded by lens L1, is reflected by mirror M and impinges the PBS. Since the direction of the beam polarization is perpendicular to the table top, it is totally reflected by the PBS and hits the BTO crystal, thus constituting the reference beam. The part of the beam transmitted through the crystal is focused by the 3-mm focal length objective lens L2 and illuminates the object; the light scattered by the diffuse object forms the object beam, as described earlier. Between the objective lens and the crystal a polarizer is positioned in order to compensate for the partial depolarization caused by the diffusely scattering surface. The holographic recording occurs by diffusion. The 28-mm focal length ocular lens L3 was positioned at a distance from the crystal which is nearly its front focal length, in order to display an enlarged holographic object image at a CMOS camera. The $8 \times 8 \times 8-\mathrm{mm}^{3} \mathrm{PBS}$ cube was placed between the ocular lens and the holographic medium. The holographic compound microscope is shown in figure 10. The size of the whole setup is $30 \times 20 \mathrm{~cm}^{2}$ approximately. This setup allows the degree of freedom to conveniently place the BTO crystal whether closer to the objective lens or to the PBS in order to get a more intense object beam, which may provide a shorter hologram buildup time. The fastest hologram recording was achieved by placing the BTO crystal at the image plane of lens L2.

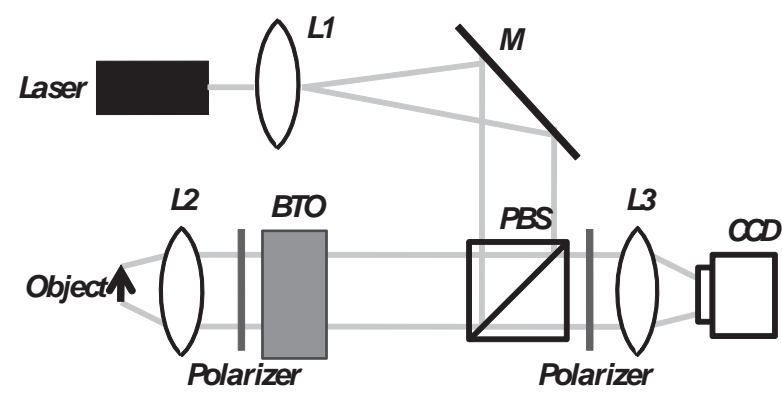

Figure 10. First configuration of the holographic compound microscope

As described in section 4.2, the crystal [001]-axis was oriented with respect to the Z-direction by setting $\gamma=|\rho d| / 2$ in order to maximize the diffracted wave intensity. Figure 11 shows the 
holographic image of a pattern chart while figure $11 \mathrm{~b}$ shows its corresponding. The distance between two neighboring bars of the chart is $100 \mu \mathrm{m}$.

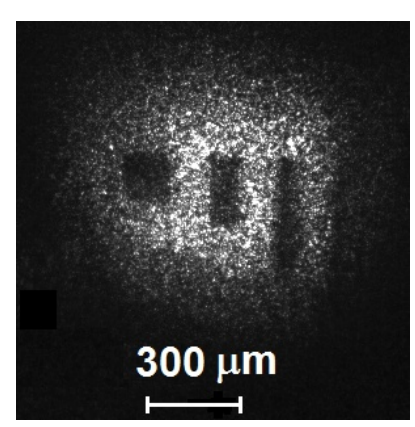

Figure 11. Holographic image of the test chart formed by the first microscope configuration

\subsubsection{Second optical configuration}

In the second microscope configuration the holographic medium is positioned between the objective lens and the object, such that the object wave at the crystal is originated directly from scaterring by the object surface, as shown in figure 12. The real holographic image built by the objective lens lays in a plane between the PBS and the ocular lens, which in turn forms the enlarged image onto the CMOS target.

This configuration requires the illuminating beam to be tightly focused onto the object surface and the crystal front face to be placed as close as possible to the object. This assures the highest possible object beam intensity inside the crystal. In addition, this small distance allows the object beam to be collected by the BTO in a very broad angular range, which increases the image resolution due to diffraction and therefore allows a higher holographic image quality. Figure 12a shows the holographic image of a chart of a 2-mm high " $x$ " letter.

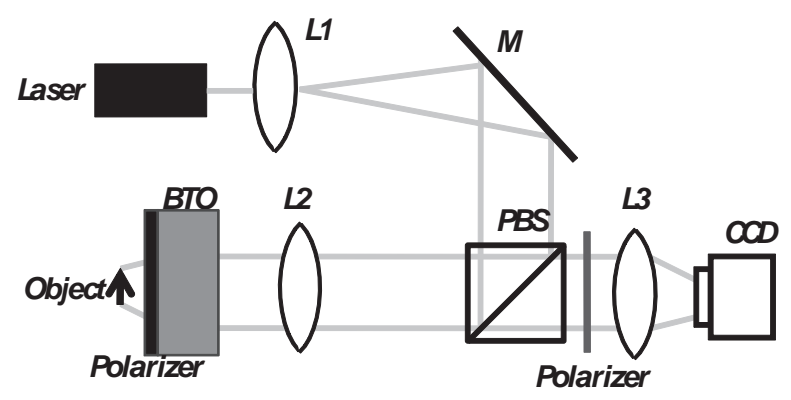

Figure 12. Second compound microscope configuration 


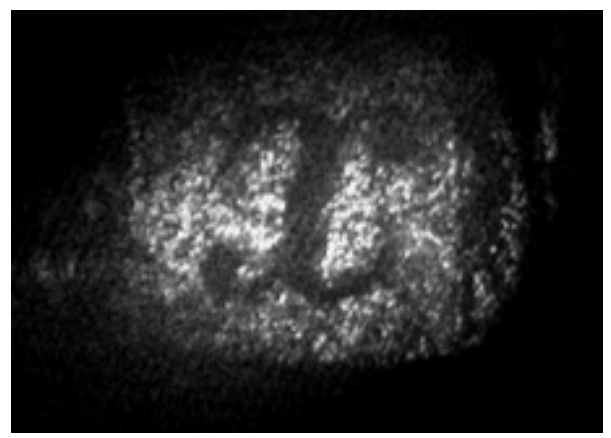

Figure 13. Holographic image built by the second optical configuration

\section{Applications}

In this section the performance of both configurations are tested and compared as well as the potentialities of the microscopes in the field of device testing and material characterization are pointed out. The most important aspects of the technique are also compared with other characterization processes.

\subsection{Microcomponent characterization by double-exposure holographic interferometry}

As a preliminary example, figure 14 shows double exposure interferograms resulting from mechanical loads. Figure 14a shows a $2.5 \times 2.5-\mathrm{mm}^{2}$ illuminated area of a flat metallic bar obtained with the first microscope configuration, while figure $14 \mathrm{~b}$ shows the chart of figure 13 submitted to a small mechanical perturbation, obtained with the second microscope geometry. In both cases the characteristic double-exposure interferograms can be visualized. Those interference patterns can be mainly attributed to a partial wave depolarization which occurs when the illuminating beam strikes the diffusely scattering object and to domains in the crystal bulk which also partially depolarize the transmitted object beam. Since part of these depolarized waves become p-polarized as they are reflected by the PBS, they interfere with the diffracted waves. While the transmitted object wavefront changes instantaneously with the perturbation, the diffracted wavefront remains unchanged in a period of the order of the hologram buildup time, so that the resulting interference between both waves generates the well-known $\cos ^{2}$-fringe pattern. By whether qualitatively or quantitatively evaluating this interferogram, the deformation map undergone by the object can be determined.

The performance of the reflection holography microscope for holographic interferometry was investigated by studying the response of a $2.96 \times 0.6-\mathrm{mm}^{2}$ a-SiC piezoresistor $(\mathrm{PZR})$ through double exposure holography. The sample was bonded to the edge of a $125 \times 25-\mathrm{mm}^{2}$ tip made of stainless steel. The side containing the PZR was fixed by a massive holder, while the free opposite side was submitted to forces applied normally to the tip. By slightly bending the plate, 


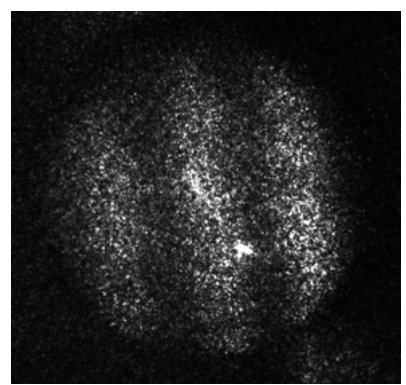

(a)

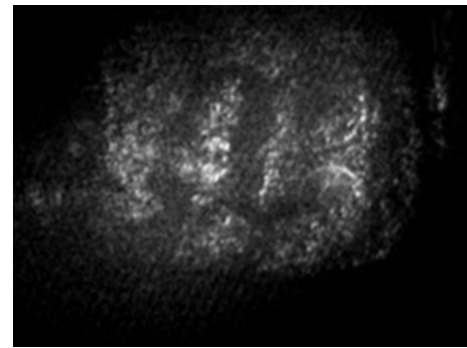

(b)

Figure 14. Double exposure interferograms obtained through: $a$ - the first and b - the second microscope configuration

typical $\cos ^{2}$-fringes are instantaneously visualized. The interference pattern results from the interference between the wave carrying the object holographic reconstruction and the partially transmitted object wave. The consequent deformation of the PZR can be noticed by examining the interferograms shown in figures $14 \mathrm{a}, \mathrm{b}$ and $\mathrm{c}$, for applied forces of $0.3,0.5$ and $0.7 \mathrm{~N}$, respectively.

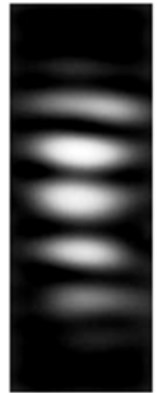

(a)

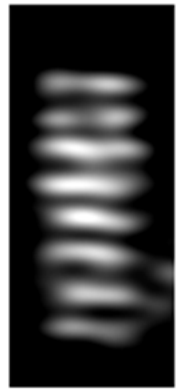

(b)

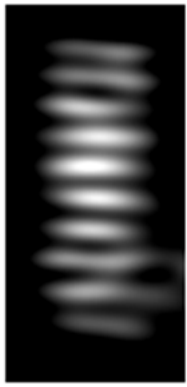

(c)

Figure 15. The deformation map of the partially bended, partially twisted PZR is shown in figure 15.

\subsection{Time average holography of a transducer}

The second configuration of the holographic microscope was also used for vibration analysis through time average holography. For this purpose a 20x15- $\mathrm{mm}^{2}$ piezoelectric ceramic and a $12.7 \times 12.7-\mathrm{mm}^{2}$ transducer were excited by dither signals. The illuminated area of the transducer is $2 \times 2 \mathrm{~mm}^{2}$. Figures $17 \mathrm{a}, 17 \mathrm{~b}$ and $17 \mathrm{c}$ show the nodal regions of the piezoelectric ceramic vibrating at $6 \mathrm{kHz}, 11 \mathrm{kHz}$ and $14 \mathrm{kHz}$, respectively; figure 18 shows the time average pattern of the piezoelectric ceramic vibrating at $1 \mathrm{kHz}$. Remarkably in figures $17 \mathrm{a}, 17 \mathrm{~b}$ and 18 the nodal regions can be clearly noticed. These brightest regions correspond to loci of zero vibration 


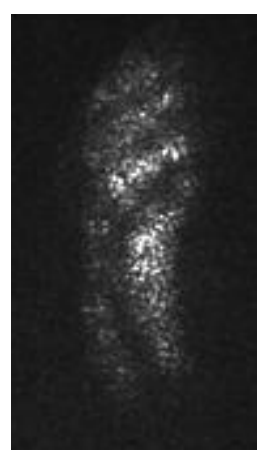

Figure 16. Double-exposure interferogram of the PZR

amplitude, as widely reported in the literature [34]. The thin dark fringes surrounding the nodal regions (denoted by " $\mathrm{P}$ " and " $\mathrm{Q}$ " in figures 17 and 18) correspond in turn to the roots of the zeroth-order Bessel function whose argument is given by $4 \pi A / \lambda$, where $A$ is the vibration amplitude and $\lambda$ is the light wavelength.

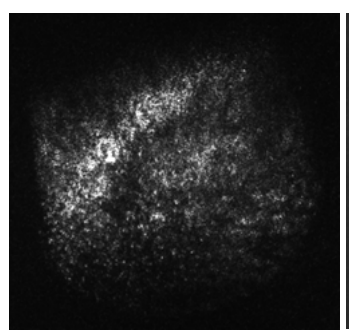

(a)

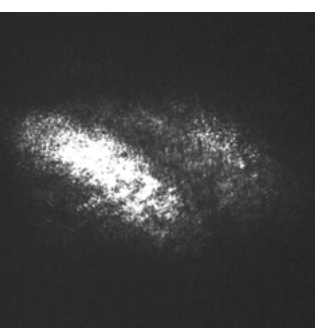

(b)

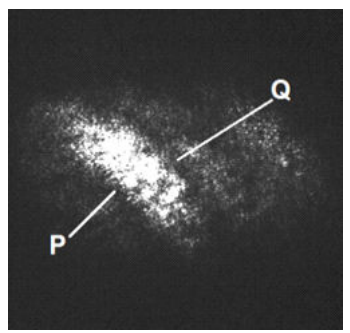

(c)

Figure 17. Time average holograms of a vibrating transducer.

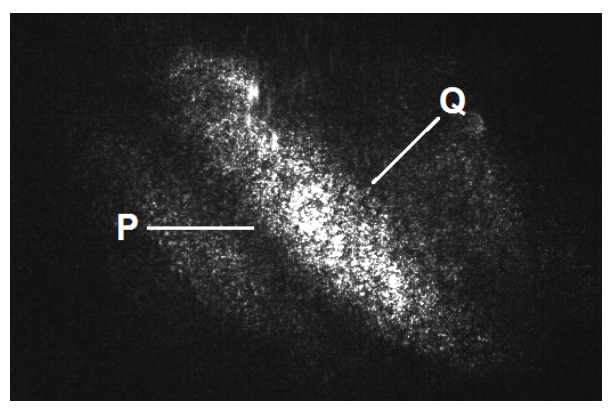

Figure 18. Time average hologram of a vibrating piezoelectric ceramic. 


\section{Conclusion}

The Denisiuk-type reflection holography microscope has been demonstrated to be very suitable for double-exposure and time average holographic interferometry. Due to the use of the PBS cube and the tilting applied to the BTO crystal, the transmitted object beam and its holographic reconstruction emerged from the crystal orthogonally polarized, thus providing high-visibility interferograms in spite of the typically low diffraction efficiency of those media. This novel conception allowed the construction of very simple and compact inline microscopes, with the objective lens working simultaneously as an illuminating lens and an imaging one. In the first optical configuration the BTO crystal is placed at the image plane of the objective, while in the second configuration the BTO was positioned between the object surface and the objective lens. Since the object wave at the holographic medium is higher in the first geometry, the hologram buildup time is shorter, as well as the diffracted wave intensity is higher, resulting in if higher-visibility interferograms, if compared to the second case. The interferograms generated in both cases points out the possibility for construction of compact and portable instruments for microscopic device characterization.

\section{Author details}

Eduardo Acedo Barbosa, Danilo Mariano da Silva and Merilyn Santos Ferreira

Laboratório de Óptica Aplicada, Faculdade de Tecnologia de São Paulo, CEETEPS - UNESP, Pça Cel Fernando Prestes, São Paulo - SP, Brazil

\section{References}

[1] Ashkin, A, Boyd, G, Dziedzic, J, Smith, R, Ballman, A, Levinstein, J, \& Nassau, K. Optically induced refractive index inhomogeneities in $\mathrm{LiNbO} 3$ and $\mathrm{LiTaO} 3$. Applied Physics Letters (1996). , 9(1), 72-74.

[2] Chen, F. La Macchia J., Fraser D. Holographic storage in Lithium Niobate. Applied Physics Letters (1968). , 13(7), 223-225.

[3] Günter, G, \& Huignard, J. Photorefractive Materials and Their Applications II. Topics Applied Physics, 62. Berlin, Heidelberg: Springer Verlag; (1989).

[4] Frejlich, J. Photorefractive Materials. New York: Wiley \& Sons; (2007).

[5] Kamshilin, A, \& Petrov, M. Continuous reconstruction of holographic interferograms through anisotropic diffraction in photorefractive crystals. Optics Communications (1985). , 53(1), 23-26. 
[6] Mallick, S. Roue'de D. Influence of the polarization direction of two-beam coupling in photorefractive Bi12SiO20: Diffusion regime. Applied Physics B: Lasers Optics (1987). , 43(4), 239-245.

[7] Huignard, J, Herriau, J, \& Valentin, T. Time average holographic interferometry with photoconductive electrooptic Bi12Si0 20 crystals. Applied Optics (1977). , 16(11), 2796-2798.

[8] Georges, M. Lemaire Ph. Phase-shifting real-time holographic interferometry that uses bismuth silicon oxide crystals. Applied Optics (1995). , 34(32), 7497-7506.

[9] Barbosa, E, \& Muramatsu, M. Mapping of vibration amplitudes by time average holography in Bi12SiO20 crystals. Optics Laser Technology (1997). , 29-359.

[10] Horwitz, B, \& Corbett, F. The PROM-theory and applications for the Pockels readout optical modulator. Optical Engineering. (1978). , 17-353.

[11] Marrakchi, A, Tanguay, A, Yu, J, \& Psaltis, D. Physical characterization of the photorefractive incoherent-to-coherent optical converter. Optical Engineering (1985). , 24-124.

[12] Mccahon, S, Kim, S, \& Tanguay, A. Optically modulated linear-array total-internalreflection spatial light modulator. Journal of the Optical Society of America A (1984).

[13] White, J, \& Yariv, A. Real-time image processing via four wave mixing in a photorefractive medium. Applied Physics Letters (1980). , 37-5.

[14] Herriau, J, \& Micheron, F. Coherent Selective Erasure of Superimposed Volume Holograms in LiNbO3. Applied Physics Letters (1975). J.P Herriau, F. Micheron, “Coherent Selective Erasure of Superimposed Volume Holograms in LiNbO3" Appl. Phys. Lett. 26, 256 (1975), 26(5), 256-258.

[15] Poon, T, \& Banerjee, P. Contemporary Optical Image Processing With Matlab, Elsevier Science (2001). , 1

[16] Feinberg, J. Real-time Edge Enhancement Using the Photorefractive effect. Optics Letters (1980). , 5(8), 330-332.

[17] Chang, C, Chen, T, Yau, H, \& Ye, P. Two-way two-dimensional pattern transferring upon requesting with BaTiO3 crystal. Optical Materials (2001). , 18(1), 103-106.

[18] Fischer, B, Cronim-golomb, M, White, J, \& Yariv, A. Passive (self-Pumped) Phase Conjugate Mirror: Theorical and Experimental Investigation. Applied Physics Letters (1982). , 41(8), 689-691.

[19] Santos, P, Faria, S, \& Tagliaferri, A. Photorefractive optical holographic correlation using a Bi12TiO20 crystal at $\lambda=0.633 \mu \mathrm{m}$. Optics Communications (1991). , 86(1), 29-33. 
[20] Alam, M, \& Khoury, J. Proceedings of the Spie Conference on Photorefractive Fiber and Crystal Devices: Materials, Optical Properties and Applications, San Diego, California (2000).

[21] Chuang, E, \& Psaltis, D. Storage of 1000 holograms with use of a dual-wavelength method. Applied Optics (1997). , 36(32), 8445-8454.

[22] Yeh, P, Khoshnevisan, M, Ewbank, M, \& Tracy, J. Possibility of relative position sensing by using double phase-conjugate resonators. Optics Communication (1986). , 57(6), 387-390.

[23] Barbosa, E, Preto, A, Silva, D, Carvalho, J, \& Morimoto, N. Denisiuk-type reflection holography display with sillenite crystals for imaging and interferometry of small objects. Optics Communications (2008). , 281(3), 408-414.

[24] Kukhtarev, N, Chen, B, Venkateswarlu, P, Salamo, G, \& Klein, M. Reflection holographic gratings in [111] cut Bi12TiO20 crystal for real time interferometry. Optics Communications (1993).

[25] Mallick, S, Miteva, M, \& Nikolova, I. Polarization properties of self-diffraction in sillenite crystals: reflection volume gratings. Journal of the Optical Society of America B (1997). , 14(5), 1179-1186.

[26] Salazar, A, Lorduy, H, Tebaldi, M, \& Bolognini, N. Analysis of reflection speckle holograms in a BSO crystal. Optics Communications (2006). , 262(2), 157-163.

[27] Salazar, A, Goez, R, Sierra, D, Garzón, J, Pérez, F, \& Lorduy, H. Optical wavelet correlator by four-wave mixing via reflection holograms in a BSO crystal. Optics Communications (2004).

[28] Erbschloe, D, Solymar, L, Takacs, J, \& Wilson, T. Two-wave mixing in reflection holograms in photorefractive materials. IEEE Journal of Quantum Electronics (1988). , 24(5), 820-826.

[29] Shepelevich, V, \& Naunyka, V. Effect of optical activity and crystal symmetry on maximal diffraction efficiency of reflection holograms in cubic photorefractive piezocrystals.Applied Physics B (2009). , 95(3), 459-466.

[30] Shandarov, S, \& Burimov, N. Kul'chin Y., Romachko R., Tolstik A., Shepelevich V. Dynamic Denisyuk holograms in cubic photorefractive crystals. Quantum Electronics (2008). , 38(11), 1059-1069.

[31] Denisyuk, Y. On the reflection of optical properties of an object in a wave field of light scattered by it. Doklady Akademii Nauk SSSR (1962). , 144(6), 1275-1278.

[32] Buse, K. Light-Induced Charge Transport Processes in Photorefractive Crystals I: Models and Methods. Applied Physics B (1997). , 64(3), 273-291. 
[33] Miridonov, S, Kamshilin, A, \& Barbosa, E. Recyclable holographic interferometer with a photorefractive crystal: optical scheme optimization. Journal of the Optical Society of America A (1994). , 11(6), 1780-1788.

[34] Hariharan, P. Optical Holography: Principles, techniques and applications. Cambridge Studies in Modern Optics, (1996). 
